\title{
Spitzer Warm Mission Transition and Operations
}

\author{
William A. Mahoney ${ }^{\mathrm{a}}$, Lisa J. Garcia ${ }^{\mathrm{a}}$, Joseph Hunt, Jr. ${ }^{\mathrm{b}}$, Douglas B. McElroy ${ }^{\mathrm{a}}$, Vince Mannings ${ }^{\mathrm{a}}$, \\ David S. Mittman ${ }^{\mathrm{b}}$, JoAnn C. O'Linger ${ }^{\mathrm{a}}$, Marc Sarrel ${ }^{\mathrm{b}}$, and Elena Scire ${ }^{\mathrm{a}}$ \\ ${ }^{a}$ Spitzer Science Center, MS 314-6, 1200 E. California Blvd., Pasadena, CA, USA 91125; \\ bJet Propulsion Laboratory, 4800 Oak Grove Dr., Pasadena, CA, USA 91109
}

\begin{abstract}
Following the successful dynamic planning and implementation of IRAC Warm Instrument Characterization activities, transition to Spitzer Warm Mission operations has gone smoothly. Operation teams procedures and processes required minimal adaptation and the overall composition of the Mission Operation System retained the same functionality it had during the Cryogenic Mission. While the warm mission scheduling has been simplified because all observations are now being made with a single instrument, several other differences have increased the complexity. The bulk of the observations executed to date have been from ten large Exploration Science programs that, combined, have more complex constraints, more observing requests, and more exo-planet observations with durations of up to 145 hours. Communication with the observatory is also becoming more challenging as the Spitzer DSN antenna allocations have been reduced from two tracking passes per day to a single pass impacting both uplink and downlink activities. While IRAC is now operating with only two channels, the data collection rate is roughly $60 \%$ of the four-channel rate leaving a somewhat higher average volume collected between the less frequent passes. Also, the maximum downlink data rate is decreasing as the distance to Spitzer increases requiring longer passes. Nevertheless, with well over $90 \%$ of the time spent on science observations, efficiency has equaled or exceeded that achieved during the cryogenic mission.
\end{abstract}

Keywords: Spitzer, spacecraft operations, observation scheduling

\section{INTRODUCTION}

The Spitzer Space Telescope ${ }^{[1]}$ is the last of NASA's four Great Observatories. It was developed for infrared astronomy using a cryogenically cooled $85 \mathrm{~cm}$ primary mirror and three instruments sensitive from 3.6 to $160 \mu \mathrm{m}$. The instruments were the Infrared Array Camera (IRAC) for photometry in four channels centered at 3.6, 4.5, 5.8, and 8.0 $\mu \mathrm{m}$, the Infrared Spectrometer (IRS) for spectroscopic measurements from 5.2-38 $\mu \mathrm{m}$ along with photometry at 16 and $22 \mu \mathrm{m}$, and the Multi-band Infrared Photometer for Spitzer (MIPS) for imaging at 24, 70, and $160 \mu \mathrm{m}$ and Spectral Energy Distribution (SED) measurements from about $50-100 \mu \mathrm{m}$. While the spacecraft operates at roughly room temperature, the instrument detector arrays and the telescope mirrors were maintained at cryogenic temperatures through a combination of radiative cooling, a superfluid helium bath, and helium boil-off gas. The observatory operated extremely well and efficiently throughout its nearly 5-year 9-month cryogenic lifetime from launch on 25 August 2003 until the helium was exhausted on 15 May 2009. Without helium, radiative cooling allowed the mirror and cryostat temperatures to stabilize at about $26 \mathrm{~K}$, too warm for operation of either IRS or MIPS, but sufficiently cold for IRAC observations at 3.6 and $4.5 \mu \mathrm{m}$. During the 10 weeks following depletion of the helium, IRAC Warm Instrument Characterization (IWIC) activities were successfully completed with the IRAC arrays now controlled at $28.7 \mathrm{~K}$ and with a performance essentially unchanged from cryogenic operations. The Spitzer Warm Mission has now been successfully running for nearly a year with a proposal pending to continue operations through the end of December 2013. Operations beyond that time, while possible, would be complicated by geometric and communication constraints.

Mission operations are being carried out by teams from the Spitzer Science Center (SSC) at Caltech, the Jet Propulsion Laboratory, and Lockheed Martin Astronautics in Denver, Colorado. The spacecraft operates autonomously, executing sequences of observations and spacecraft activities typically packaged and uplinked in 1-week segments. Observational efficiency has been excellent, averaging well over $90 \%$ through the warm mission to date. Spitzer executed over 36,000 hours of scientific observations during the cryogenic phase and is continuing in the warm mission at a rate approaching 8000 hours/year.

Observatory Operations: Strategies, Processes, and Systems III, edited by

David R. Silva, Alison B. Peck, B. Thomas Soifer, Proc. of SPIE Vol. 7737, 77371W

(c) 2010 SPIE $\cdot$ CCC code: $0277-786 X / 10 / \$ 18 \cdot$ doi: $10.1117 / 12.857814$

Proc. of SPIE Vol. 7737 77371W-1 


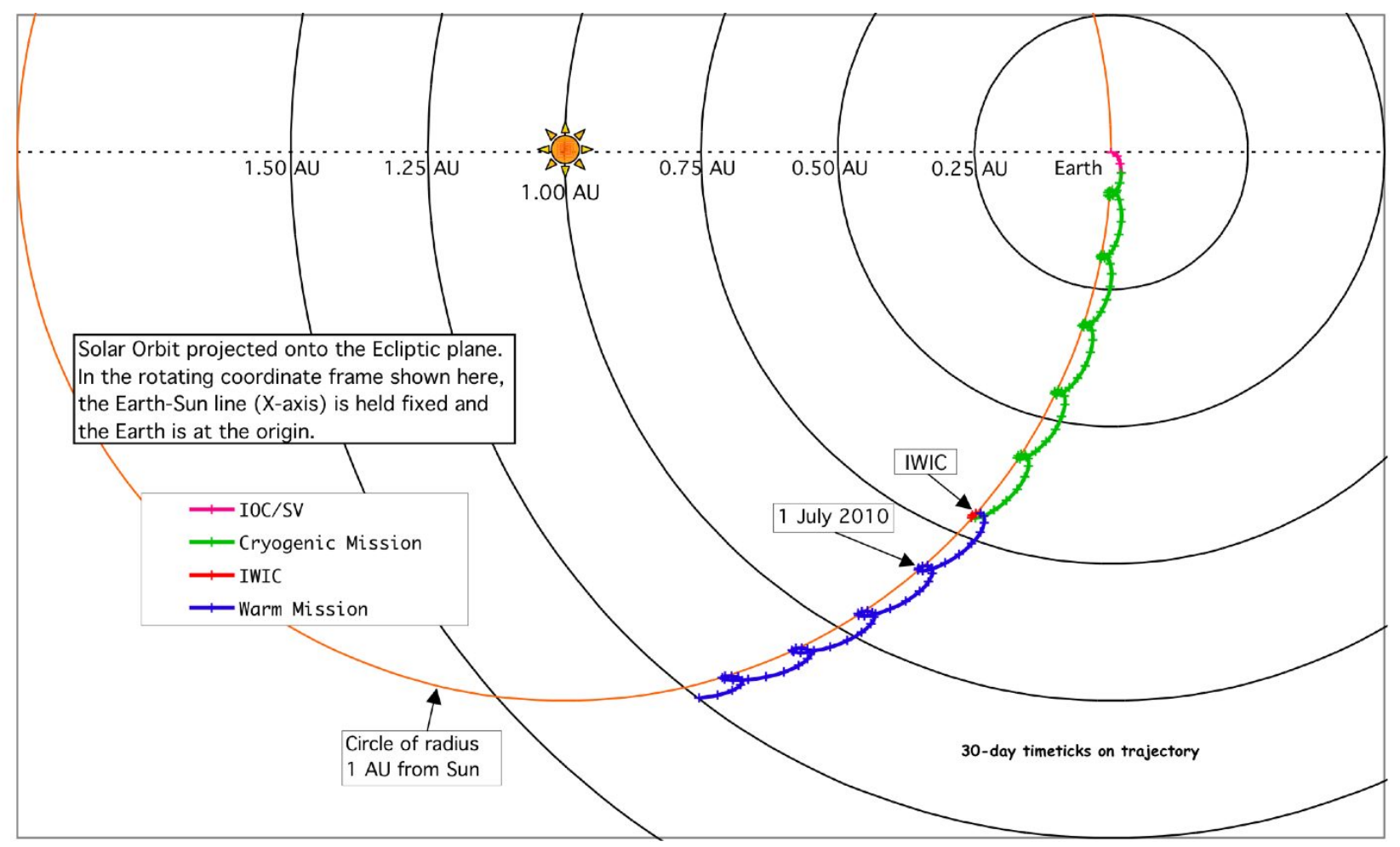

Figure 1: The Spitzer orbit as viewed from the ecliptic North with the Sun toward the left. The loops in the orbit occur at approximately one-year intervals when Spitzer is at perihelion. If approved, the warm mission will extend through 31 December 2013 at which time the spacecraft will be 1.25 AU from Earth.

\section{GENERAL OPERATIONS AND MISSION PHASES}

Spitzer is in a unique Earth-trailing solar orbit ${ }^{[2]}$, receding from Earth at the rate of about $0.12 \mathrm{AU}$ per year and currently at a distance of about $0.8 \mathrm{AU}$ (Figure 1). This orbit has the great advantage of providing a very stable thermal environment while significantly simplifying operations compared to Earth orbiting observatories that must contend with Earth eclipses and a bright Moon. The most significant disadvantage of the orbit is the complexity of telecommunications as the signal levels decrease with increasing distance from Earth. The spacecraft is also more susceptible to damage from solar proton flares without the radiation shielding of the van Allen belts.

The basic scientific activity is the Astronomical Observational Request (AOR) that is defined by the observer using an Astronomical Observation Template (AOT), a Java client application with menus the user fills out to define a particular observation. Each AOR is converted directly into a series of spacecraft commands, with AORs having durations ranging from a few minutes up to 8 hours during the cryogenic phase, depending on the instrument and the mode of operation. For the warm mission the maximum AOR duration was extended to 24 hours. In a limited number of cases, AORs have been converted into Instrument Engineering Requests (IERs) to reduce data volumes by deleting non-prime fields and to enable observations longer than the AOR maximum of 24 hours. Such IERs have been used primarily for very long, continuous observations of extra-solar planets (exo-planets). The longest continuous observation executed to date was 70 hours, about the maximum possible without risking a spacecraft anomaly. A 145-hour observation of HD147506 was carried out with two intervening downlinks.

The observatory operates autonomously between contacts with the Deep Space Network (DSN) that during the cryogenic phase were typically scheduled twice per day. During the warm mission DSN contacts have been reduced to one per day with a current maximum downlink rate of $1.65 \mathrm{Mbps}$ and with an average daily volume of just under 1 GByte, including spacecraft and instrument engineering data. The AORs, together with instrument calibrations and spacecraft activities, are packaged together into a series of activities covering each Period of Autonomous Operations (PAO - the time between Earth contacts). During the cryogenic mission only one instrument was operated at a time to avoid over taxing the spacecraft power system and to avoid generating data volumes in excess of the on-board storage 
capacity. During the Warm Mission IRAC is powered on continuously. A room temperature star tracker determines the spacecraft pointing by averaging the positions of about 35 stars selected from an on-board star catalog. The star tracker axis is aligned with the telescope boresight as defined by two Pointing Control Reference Sensors (PCRS) near the IRAC arrays. Offsets between the star tracker and the telescope boresight are measured twice per day to reduce small cryomechanical variations in pointing. The star tracker can be used to directly point the telescope with an accuracy of about 0.4 arcsec rms radial $(1 \sigma)$.
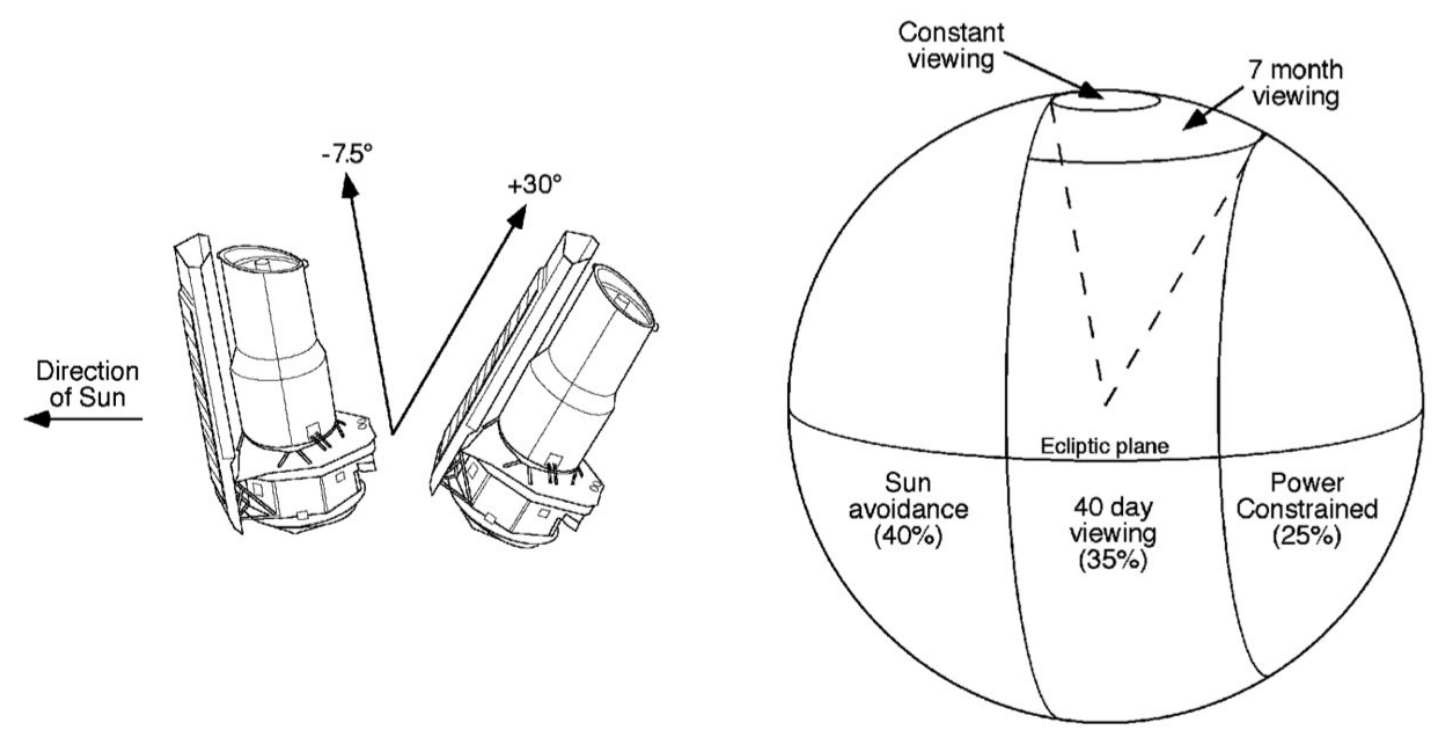

Figure 2. Due to spacecraft thermal and power constraints and the need to avoid the Sun, the telescope boresight must always point roughly perpendicular to the Sun leaving an operational pointing zone of about 1/3 of the sky.

The solar panels, which thermally shield the telescope, point roughly toward the Sun with the telescope boresight oriented perpendicular to the solar panels (Figure 2). For solar avoidance and to meet spacecraft thermal and power constraints, the boresight is allowed to point no closer than 82.5 degrees from the Sun and no further than 120 degrees, thus restricting observations to an Observational Pointing Zone (OPZ) which covers about $35 \%$ of the sky at any given time. Sources near the ecliptic plane are in the OPZ for about 40 days twice per year while sources near an ecliptic pole are continuously visible. The only expendable resource on board now is pressurized nitrogen gas used by the reaction control system to remove angular momentum that builds up as the result of solar radiation pressure. Opportunities for momentum de-saturation are scheduled twice daily, usually as part of the downlink process. There is sufficient nitrogen remaining to last well beyond any reasonable extension of the Spitzer mission.

\subsection{Mission Phases}

The mission can be broken into several distinct operational phases (Table 1). Following launch the observatory entered a 3-month In-orbit Checkout/Science Verification (IOC/SV) period during which the spacecraft systems and the three instruments were activated and characterized and optimization of their performance was initiated. In IOC special teams were established for the planning and execution of activities that were not part of the nominal operation phase. In contrast to the IRAC Warm Instrument Characterization (IWIC) phase, three instruments had to be managed instead of one. Some [most of IWIC was carried out off Earth-point doing calibration and science observations] of the IOC/SV activities were conducted with the observatory at a fixed attitude to enable constant communication. There were no limiting factors for ground communication resources because the telecommunications link margin was not an issue.

Following IOC/SV the observatory began the primary mission that consisted of nearly $5 \frac{1}{2}$ years of routine scientific observations. This period was broken into instrument campaigns of duration typically 1 to 3 weeks during which each of the three instruments was operated, typically cycling from IRAC to MIPS to IRS. The timing and duration of the campaigns were determined by the observational constraints and the relative time required for observations with each instrument. During the first several months of the prime mission, observing efficiency was significantly improved through optimization of systems and processes including improvements to the slew model and in the instrument observational modes. 
Table 1: Mission phases.

\begin{tabular}{|l|l|}
\hline Phase & Start and End Dates \\
\hline Launch & $2003-08-25$ \\
\hline IOC and SV & $2003-08-25 / 2003-12-01$ \\
\hline Cryogenic Mission & $2003-12-01 / 2009-05-15$ \\
\hline Standby caused by end of helium & $2009-05-15$ at 2211 UT \\
\hline IWIC & $2009-05-16 / 2009-07-27$ \\
\hline Proposed Warm Mission & $2009-07-27 / 2013-12-31$ \\
\hline
\end{tabular}

Throughout the prime mission helium usage was monitored and it's lifetime modeled. A number of steps were taken to extend the lifetime including actively cooling the mirrors only for MIPS observations and scheduling mainly IRS observations during the last couple of months. The thermal modeling proved exceptionally accurate and during the last two years the predicted date for cryogen exhaustion remained constant at April 2009. However, to be very conservative, it was assumed that this date was uncertain by as much as three months. Thus the plans and preparations for IWIC were based on the helium running out in January 2009.

When the helium was exhausted on 15 May 2009, the observatory entered its standby mode as expected when a number of the cryostat temperatures exceeded their allowable limits. This initiated the IWIC phase with the purpose of characterizing and calibrating the IRAC instrument at the warmer operating temperature. No changes in spacecraft performance were expected at the warmer temperature, and none were observed. Therefore only routine spacecraft maintenance activities were executed during IWIC. It was anticipated that this phase would last about 6 weeks, however, an anomaly in the IRAC array temperature control logic caused this phase to be extended by about a month. Since the successful completion of IWIC on July 27, 2009, the observatory has been conducting routine warm mission operations with IRAC on continuously.

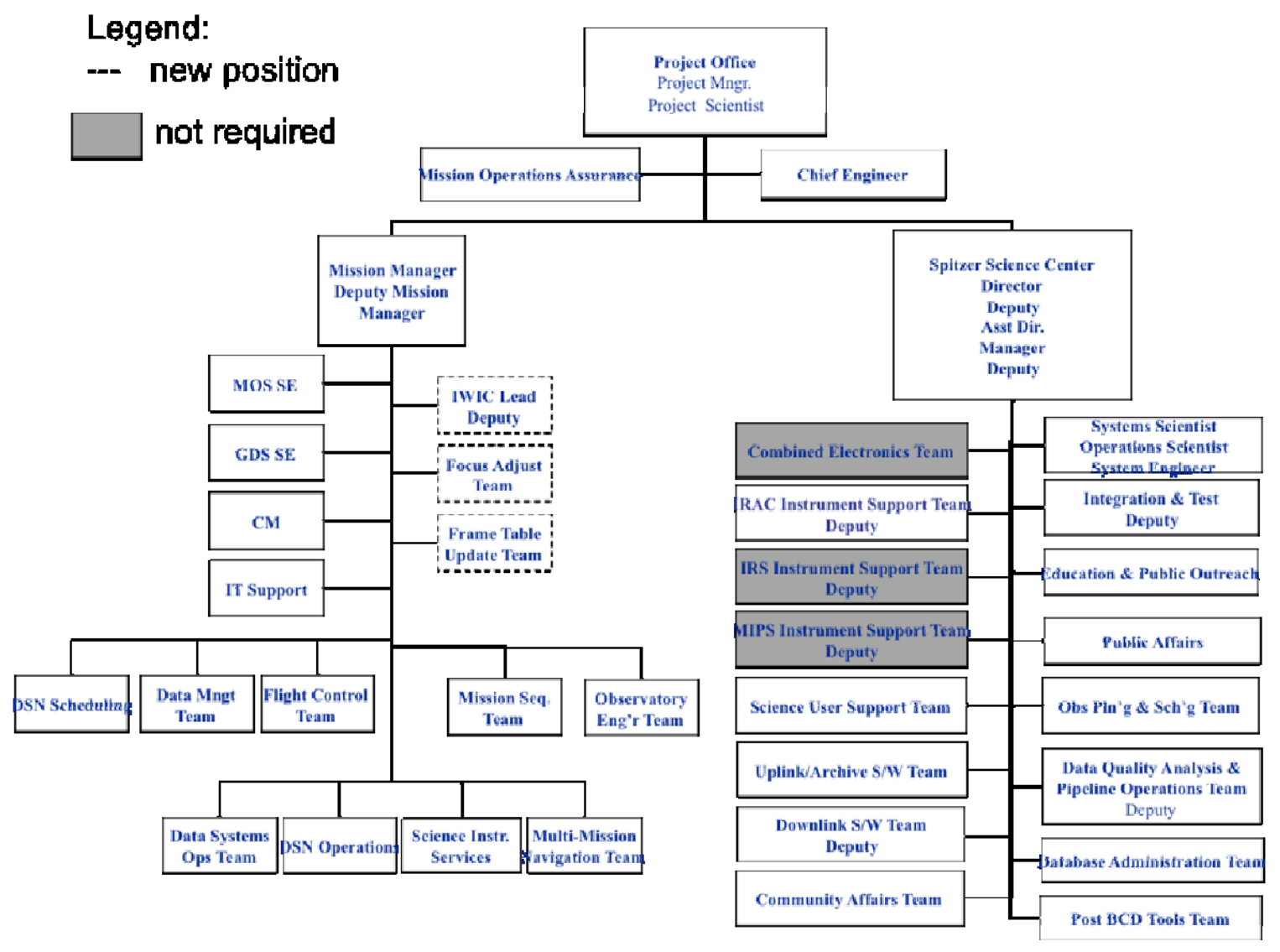

Figure 3. Spitzer Mission Operation System with changes for IWIC noted. 


\subsection{MOS Organization}

The Spitzer Mission Operation System (MOS) consists of flight and ground segments, where each include hardware and software components ${ }^{[3]}$. The ground system is composed of the MOS (Figure 3) and the Ground Data System (GDS). The MOS contains the people, teams, processes and procedures used to operate the mission. The GDS contains the ground data system hardware and software and includes not only computers and networks, but distributed physical facilities like the mission support areas, the science center, and multi-mission facilities.

The IWIC operational organization remained the same as that during cryogenic operations except for the addition of the IWIC Lead and deputy who were responsible for planning, coordinating, and tracking MOS activities across teams, and the elimination of the IRS, MIPS, and related Combined Electronics (CE) teams. While not expected to be needed, Focus Adjust and Frame Table Update teams were standing by in the unlikely event that the telescope focus was found to be out of specification or that the IRAC pointing frame definitions required changes.

\section{IWIC AND TRANSITION TO WARM MISSION}

\subsection{Planning and Requirements}

The IRAC warm instrument characterization consisted of a set of on-orbit tests and observations with five primary objectives: (a) to check the functionality of IRAC near $30 \mathrm{~K}$ where the IRAC baseplate was expected to stabilize, (b) to determine the best operating parameters (array biases, temperature set points, observation frame times and Fowler numbers), (c) to characterize properties of the data such as noise and artifacts, (d) to collect calibration data to determine the dark bias, flat-field, linearity and other required information to convert from raw data number to flux-calibrated images in units of milliJansky per steradian $(\mathrm{mJy} / \mathrm{sr})$, and (e) to perform representative science observations to inform the observing community of the performance of the warm IRAC instrument.

The IWIC driving requirements, structured to establish the baseline functionality for collecting science with the IRAC instrument operating at approximately $30 \mathrm{~K}$, were:

- Verify that all observatory subsystems are nominal,

- Verify that warm IRAC can take data,

- Set the operating parameters in the data taking blocks to support nominal operation of the warm IRAC,

- Determine that the data taken with the warm instrument, after deployment of the updated sci2 block library, can be calibrated.

Planning for IWIC was carried out by the MOS teams, through a series of Mission Operations System Design Team (MOSDT) meetings. Some engineering decisions that supported the development of this plan were made at Flight Engineering Team (FET) meetings. The IWIC planning included detailed timeline walkthroughs and a pre-build, review, and approval of 53 mini sequences as they were expected to be executed. The walkthroughs were a series of table-top exercises designed to validate the activity flow from the anomaly response, through redesign and execution of the prebuilt mini sequences once their execution time was determined, and finally sequencing for the transition to the nominal warm mission. Implementation and schedule drivers were known to include uncertain telecommunications allocations, concern about latent images on the arrays, and block library updates. All MOS teams participated in the table-top meetings and were useful in identifying areas for improvement in the plan.

In December 2008, we published an IWIC Plan that consolidated the MOS planning into a formal document. A formal review, conducted in January 2009, gave full concurrence to proceed with the plan as written. A series of schedules were developed to layout the work needed to plan and execute IWIC activities.

The original IWIC plan design goals were to be as responsive as possible to late changes from the IRAC team during IWIC sequence development. For implementation of this, the MOS design required:

- Sequence length of 12 hours vs. the nominal 7 days,

- Short lead time for building sequences (2 to 3 days),

- For unique cases the sequence development cycle was less than 2 days,

- Teams working two shifts per day (prime and second),

- Build the first Nominal Warm mission sequences on compressed schedule during IWIC,

- Mini sequences were built to execute in relative time rather than the standard absolute time.

The sequence products for the majority of activities for IWIC were built and tested in advance to validate the build process and minimize the workload during IWIC. These sequences were built and tested in parallel with the on-going weekly cryogenic sequence development and were designed in close coordination with the IRAC instrument team that 
defined both the content and the order of the IWIC activities. The only types of work anticipated during IWIC itself were changes to the mini-sequence length to fit the telecommunications allocation, updated targets because the original targets may no longer be in the OPZ, and changing the IRAC instrument settings based on results from previous minisequences. To maximize our flexibility in scheduling and executing these sequences, starting January 1, 2009, the DSN allocations consisted of two 4-hour 70-meter/34-meter antenna array tracks per day. A significant concern during IWIC planning was the unknown response of the IRAC arrays to bright sources that had caused serious latent images at cryogenic temperatures. Therefore a significant effort was expended to minimize the time the telescope might be pointing at a bright object. The mini-sequences typically contained about 8 hours of IWIC activities followed by 8 hours of buffer AORs that pointed the telescope toward a dark region. The plan for the final sequencing was to simply reduce the buffer time such that the sequences fit correctly between the DSN allocations. For a more detailed discussion of IWIC planning and execution, see Hunt, et al. ${ }^{[4]}$.

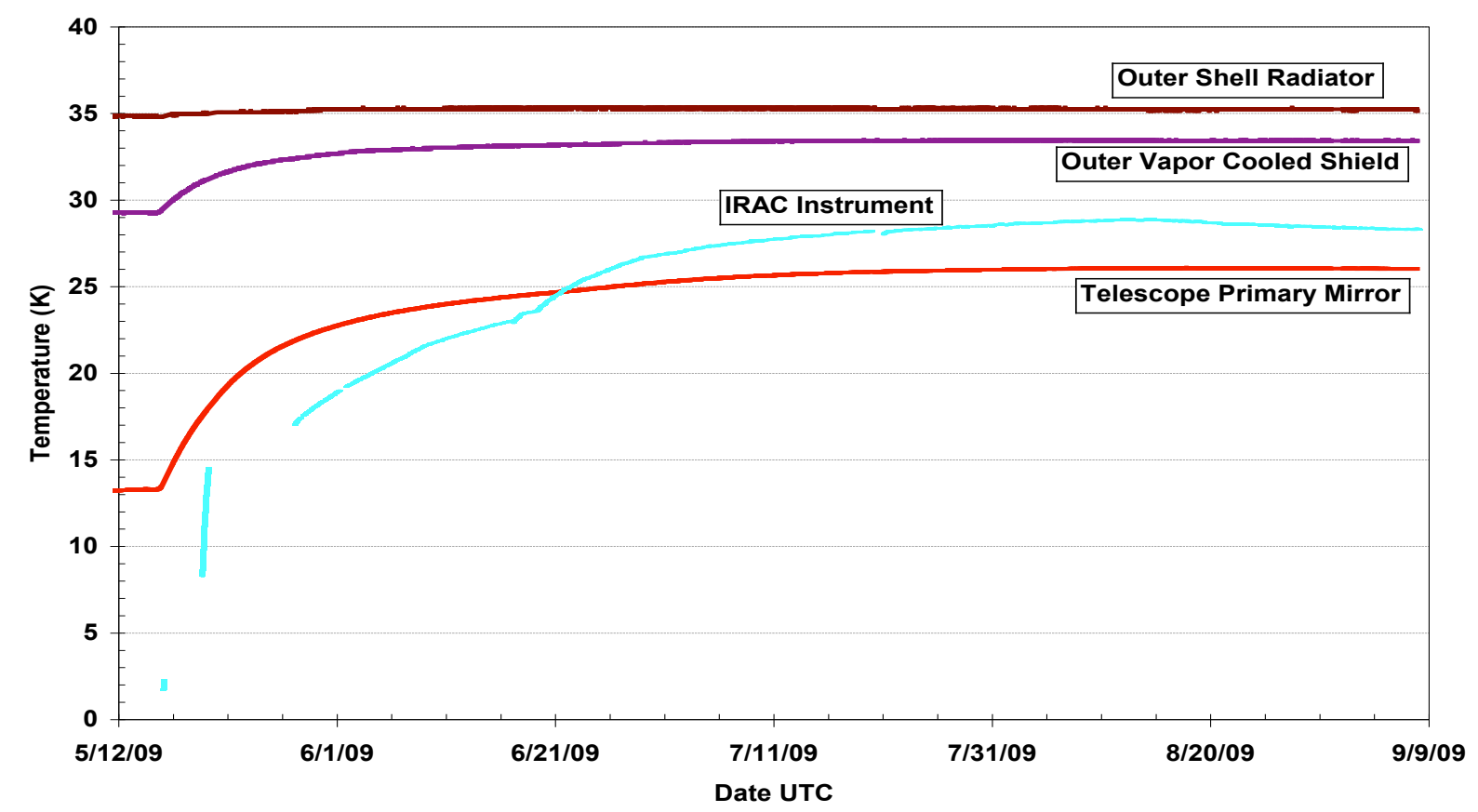

Figure 4. Temperature profiles of IRAC and the primary mirror from just before helium depletion on 15 May 2009 until stable warm mission temperatures were reached in the fall of 2009.

\subsection{Anomaly}

The first IWIC mini-sequence began as planned about 3 days after the cryogen exhaustion, following recovery from the standby mode and initial spacecraft checkout. However, immediately after the downlink of telemetry from that minisequence, we discovered that the IRAC arrays were operating at a higher temperature than expected (see rapid rise in IRAC temperature on 19 May 2009, shown in Figure 4). The IRAC array temperatures could not be controlled at the higher temperatures planned for the warm mission. It was found that the digital-to-analog converter used to convert the commanded temperature set point was physically only twelve bits wide, while the documentation and software on the ground specified sixteen bits. This problem was not discovered during the cryogenic mission because truncation of the 4 most significant bits did not change the commanded level but they were required to control the array temperatures near $30 \mathrm{~K}$.

The solution required an IRAC flight software patch that took about one month to design, develop, test, and implement. During this time the telescope and IRAC arrays continued to slowly warm. While the array temperatures could not be actively controlled during much of this time, the temperature rise was slow enough and remained sufficiently stable to allow characterization activities to continue and to carry out a very deep observation of the most distant cosmic gamma-ray burst (GRB) ever seen. 


\subsection{Execution}

Following recovery from the anomaly, six 12-hour mini-sequences were executed as originally planned. For subsequent mini-sequences the bulk of the individual activities remained valid but the sequence structure and content did not. Following the first 3 days of the resumption of IWIC, the sequence durations were expanded to 24 hours with one of 48 hours. The longer durations not only reduced the stress on the sequencing development teams and reduced the work shifts for some of the MOS teams, but also added considerably to the efficiency allowing partial recovery from the time lost to the anomaly. Very little time was expended pointing at the dark buffer region. The trade-off for the reduced work shift strategy and longer mini-sequences was an extended response time in implementing changes.

However, near the end of the planned IWIC activities it was determined that the IRAC arrays were being operated at too high a temperature and that the heat dissipated to control their temperature had caused the IRAC baseplate temperature to rise slightly above its equilibrium level (Figure 4). As the array performance is a very strong function of temperature, toward the end of IWIC it was found that the operating temperature of the arrays could be dropped from $31.0 \mathrm{~K}$ to 28.7 $\mathrm{K}$. Since the IRAC arrays had already been calibrated at the higher temperature, this change required an 8-day recalibration of IRAC that was carried out two months into the warm mission.

As expected, the telescope remained in focus and no update of the frame table was required. Unexpectedly, the latent image effects from bright sources were greatly reduced from those experienced at the lower temperatures of the cryogenic mission ${ }^{[5]}$.

\subsection{Transition to Warm Mission}

Most of IWIC was carried out using relative time master sequences whose execution began as soon as the sequence was radiated to the observatory. By contrast, routine operations are controlled by absolute-timed master sequences with typical durations of 1 week. Furthermore, any observations of moving targets (comets, asteroids, etc) must be done with absolution time masters to assure proper timing. Thus to complete IWIC and to prepare for the smooth transition to routine warm mission operations, the last 5 days of IWIC were built as an absolute time master sequence.

In parallel with the last half of IWIC, the first weeks of the warm mission were built on a highly accelerated schedule. To ease the workload, observations from several Exploration Science programs had been identified as easy to schedule having minimal observational constraints and relatively low data volumes. SSC teams worked with the program PIs who were able to quickly re-plan their observations, when necessary, for the time when routine operations began. As the performance of IRAC became better understood and calibrated over the first several months of the warm mission, additional programs were released for scheduling.

\section{WARM MISSION OPERATIONS AND SCHEDULING CHALLENGES}

\subsection{Scheduling}

The warm mission is being run much the same as the prime cryogenic mission except that IRAC is on continuously and observing only with the 3.6 and $4.5 \mu \mathrm{m}$ channels. Other than those associated with IRS and MIPS, all the operations teams, processes, and tools remain the same. Sequences continue to be built, processed, and executed in 1-week segments. Scheduling observatory activities remains the responsibility of the SSC and is carried out by the Observatory Planning and Scheduling Team (OPST). It involves two major components: (a) long range planning during which issues are identified and resolved, often through discussions with the investigator teams, and provisional times are identified for the more difficult and highly constrained observations and (b) short term scheduling when the specific weekly sequences are designed, reviewed, and prepared for execution on the observatory (see Mahoney et al. ${ }^{[6]}$ ).

Long Range Planning starts with the call to the scientific community to submit proposals for observations using Spitzer. There is typically one proposal call per year, however, a special solicitation was issued in 2008 calling for large, Exploration Science (ES), programs to be executed during the first two years of the warm mission. Following the peer review and selection process, the approved observations were added to the SSC database and made available for scheduling in December 2008, consistent with the IWIC schedule and warm mission operations even for the earliest reasonable time that the helium would run out. As a result of this call, 10 programs with a total of 10345 hours were selected (Table 2). Two additional programs were recommended by the review panel and approved as part of the SSC Director's Discretionary Time (DDT) allotment. Together these programs include both extragalactic and galactic studies with numerous observations of time variable objects and exo-planets ${ }^{[7]}$. The Spitzer Cycle 6 call for smaller 
programs, to be executed during the first year of the warm mission, led to the selection of an additional 1550 hours of observations that were made available for scheduling by the end of April 2009. The warm mission proposal process is similar to that used during the cryogenic mission except that the number of accepted proposals has been limited to reduce the expense of reviewing, monitoring, and funding a large number of small programs. Once proposals are received at the SSC, they undergo a review for both technical feasibility and scheduling difficulty. Any issues identified are passed on to the proposal review panels as an aid in their selection process.

Table 2. Exploration Science (ES) programs approved for the first two years of warm mission operations.

\begin{tabular}{|c|c|c|c|c|c|}
\hline PID & $\begin{array}{c}\text { Science } \\
\text { Category }\end{array}$ & $\begin{array}{l}\text { PI } \\
\text { Institution }\end{array}$ & Title & Co-Is & Hours \\
\hline 60010 & cosmology & $\begin{array}{l}\text { Wendy Freedman } \\
\text { Carnegie Observatories }\end{array}$ & The Hubble Constant & 5 & 705 \\
\hline 60022 & $\begin{array}{c}\text { high-z } \\
\text { galaxies }\end{array}$ & $\begin{array}{l}\text { Giovanni Fazio } \\
\text { Smithsonian Astrophysical Obs. }\end{array}$ & SEDS: The Spitzer Extended Deep Survey & 46 & 2108 \\
\hline 60024 & $\begin{array}{l}\text { high-z } \\
\text { galaxies }\end{array}$ & $\begin{array}{l}\text { Mark Lacy } \\
\text { National Radio Astronomy Obs. }\end{array}$ & $\begin{array}{l}\text { SERVS: the Spitzer Extragalactic } \\
\text { Representative Volume Survey }\end{array}$ & 46 & 1400 \\
\hline 60034 & $\begin{array}{l}\text { high-z } \\
\text { galaxies }\end{array}$ & \begin{tabular}{|l|} 
Eiichi Egami \\
University of Arizona \\
\end{tabular} & $\begin{array}{l}\text { The IRAC Lensing Survey: } \\
\text { Achieving JWST depth with Spitzer }\end{array}$ & 12 & 526.4 \\
\hline 60007 & $\begin{array}{l}\text { nearby } \\
\text { galaxies }\end{array}$ & $\begin{array}{l}\text { Kartik Sheth } \\
\text { National Radio Astronomy Obs. }\end{array}$ & $\begin{array}{l}\text { The Spitzer Survey of Stellar Structure in } \\
\text { Galaxies (S4G) }\end{array}$ & 29 & 637.2 \\
\hline 60021 & exoplanets & $\begin{array}{l}\text { Heather Knutson } \\
\text { U. C. Berkeley }\end{array}$ & $\begin{array}{l}\text { Dynamic Studies of Exoplanet Atmospheres: } \\
\text { From Global Properties to Local Physics }\end{array}$ & 10 & 1138 \\
\hline 60028 & exoplanets & $\begin{array}{l}\text { David Charbonneau } \\
\text { Harvard University }\end{array}$ & $\begin{array}{l}\text { Confirmation and Characterization of Kepler Mission } \\
\text { Exoplanets: The Era of Rock and Ice Exoplanets }\end{array}$ & 9 & 800 \\
\hline 60020 & $\begin{array}{l}\text { galactic } \\
\text { structure }\end{array}$ & $\begin{array}{l}\text { Barbara Whitney, SSI } \\
\text { Space Science Institute }\end{array}$ & $\begin{array}{l}\text { GLIMPSE360: Completing the Spitzer Galactic } \\
\text { Plane Survey }\end{array}$ & 51 & 1980.3 \\
\hline 60014 & $\begin{array}{c}\text { young } \\
\text { stellar obj. }\end{array}$ & \begin{tabular}{|l} 
John Stauffer \\
Spitzer Science Center
\end{tabular} & $\begin{array}{l}\text { Young Stellar Object Variability: Mid Infrared Clues to } \\
\text { Accretion Disk Physics \& Protostar Rotational Evolution }\end{array}$ & 36 & 550 \\
\hline 60012 & $\begin{array}{c}\text { near-earth } \\
\text { objects }\end{array}$ & \begin{tabular}{|l|} 
David Trilling \\
Northern Arizona University
\end{tabular} & $\begin{array}{l}\text { The Warm Spitzer NEO Survey: Exploring the history } \\
\text { of the inner Solar System and near Earth space }\end{array}$ & 14 & 500 \\
\hline 60003 & exoplanets & $\begin{array}{l}\text { Joseph Harrington } \\
\text { University of Central Florida }\end{array}$ & The Spitzer Exoplanetary Atmosphere Survey & 16 & $\begin{array}{c}200 \\
\text { DDT }\end{array}$ \\
\hline 60027 & exoplanets & $\begin{array}{l}\text { Michael Gillon } \\
\text { Geneva University }\end{array}$ & Detecting the Transits of Nearby Super-Earths & 12 & $\begin{array}{c}100 \\
\text { DDT }\end{array}$ \\
\hline
\end{tabular}

Once a program is approved, its AORs are entered into the Spitzer Science Operations Database (SODB) along with resource estimates such as the AOR durations, estimated slew and settling times, and estimated uplink and downlink data volumes. AORs with their associated resource estimates are a fundamental input to SIRPASS (Spitzer Integrated Planning and Scheduling Subsystem), the primary tool used by OPST for scheduling. A more detailed description of the heritage and features of SIRPASS and some related scheduling tools has been given by Barba, et al ${ }^{[8]}$. SIRPASS is a user interactive application that provides the scheduler a platform for developing the schedule, assessing numerous scheduling options and requirements, and providing a number of reports, many of which flag scheduling errors. SIRPASS uses system resources such as the DSN allocation file while enforcing constraints such as assuring pointing remains within the OPZ and honoring user-imposed constraints on the AORs. It includes the Spitzer slew model and the PCRS star catalogue and it supports a formal constraint language as well as weighting factors to facilitate early scheduling of the higher priority observations. Elements of SIRPASS are used for both long range planning and short term scheduling.

When selected programs are available, OPST starts planning for those with special requirements. For the warm mission such programs include long observations of exo-planets (there are 13 approved observations with durations ranging from 63 to 145 hours with a number of others exceeding 24 hours), highly constrained programs, observations with very high data volumes, and large maps to be executed over short time periods. For unique situations, especially the long exoplanet observations, OPST must negotiate DSN tracks consistent with the observation.

With only channels 1 and 2, the average IRAC data collection rate is now about $60 \%$ of the 4 -channel rate. During the prime mission downlinks were scheduled twice per day but for the warm mission this has been reduced to once per day 
with the goal of keeping the intervals at 24 hours to aid in scheduling work shifts for mission controllers. Thus on a perPAO basis, the average IRAC data volumes are higher than experienced during the cryogenic mission. There are also new integration times that lead to even higher data accumulation rates than were possible during the cryogenic mission. These changes place additional stress on the scheduling process to avoid overloading the on board Mass Memory Card (MMC). While there were periods during the prime mission when the data collection rate was low enough to allow skipping scheduled tracks, this is rarely the case in the warm mission except during long exo-planet observations. Management of the data stored in the $\mathrm{MMC}^{[9]}$ is the responsibility of JPL; however, it can cause a significant complication to the scheduling process. OPST must design sequences such that the bandwidth for data playback for each pass is sufficient and that the risk of a missed pass (e.g. for weather or antenna problems) is sufficiently mitigated.

Short Term Scheduling starts approximately six weeks before the sequence begins execution on the observatory. It is expected that, with the exception of high-impact Targets of Opportunity (ToOs), all potential observations for that week have been finalized and delivered to the SODB along with the standard instrument calibrations and spacecraft activities. The IRAC instrument support team provides all instrument calibration requests and scheduling instructions.

The weekly build starts with the downlinks as defined in the DSN station allocation file provided by JPL, followed by the spacecraft activities, including the PCRS/star tracker alignment measurements and momentum removal opportunities every 12 hours. While IRAC is now on continuously, time is still broken into 2-week campaigns for calibration deliveries and for data reprocessing and archival. A standard set of darks, flats, and calibration stars is scheduled at the beginning and middle of each campaign, typically during the first PAO of each week. Often the long range planning has identified a number of observations (e.g. exo-planets, large maps, and constrained observations from previous weeks) that must be included. The remaining time is usually filled using an algorithm that selects observations from the pool of available AORs and attempts to efficiently fill the gaps by minimizing slew time while factoring in AOR priorities, honoring all formal constraints, and verifying pointing is within the OPZ.

Even during the cryogenic mission, IRAC observations were typically the most highly constrained and difficult to schedule. This problem is exacerbated in the warm mission because the number of exo-planet observations has greatly increased and there are several programs carrying out variability studies that can each consist of hundreds of AORs, with some having nearly 100 highly constrained follow-on observations. Furthermore, several Exploration Science programs (Table 2) consist of thousands of AORs, a number that far exceeds even the larger cryogenic programs and that increases processing and management times.

The management of data volumes is also more challenging for warm mission scheduling. The data collection rate can vary by nearly an order of magnitude, depending on the observing mode and the integration times. Large maps tend to have large data volumes because of short integration times. These must often be interleaved with deep observations that use longer integration times in order to meet data volume requirements. In addition, the channel 2 images using several short integration times compress very poorly and the scheduling process has recently been modified to more accurately estimate these data volumes. Once the basic structure of the week is set, a significant effort must be expended to assure that all required observations are included, observational constraints are honored, the IRAC calibrations are protected from bright source latent images, and the efficiency is at a practical maximum. Considerable shuffling is usually required to meet these requirements, to minimize slew time, and to optimize the telecommunication sessions within the DSN station allocation times. The complexity of some weeks can lead to unavoidable constraint violations, especially when the pool of available observations is running low.

The SIRPASS scheduling reports are then distributed to the IRAC instrument team, the SSC Science User Support Team, JPL Mission Operation teams, and the Observatory Engineering Team for review and approval. After approval, the week is packaged and the Pass 1 sequence products are delivered to the JPL Mission Sequence Team (MST) where they are run through extensive modeling, checked for flight rule violations, and distributed for final review and approval. Errors found in the modeled products can require an OPST re-build and a Pass 2 delivery of the corrected sequence. After the products pass the review cycles the MST conducts a command conference to coordinate the uplink of the command products to the observatory.

Despite the scheduling challenges, the Spitzer observational efficiency has remained exceptionally high. Figure 5 shows weekly averages of the fractional time spent on various activities. From the bottom up they are (a) science observations, (b) instrument calibrations, and (c) initial slews to the science target. These three together define the efficiency that has averaged well over $90 \%$ for the warm mission to date. There have been no anomalies or scheduling conflicts and the gap and slave halt times are too short to see in Figure 5. 


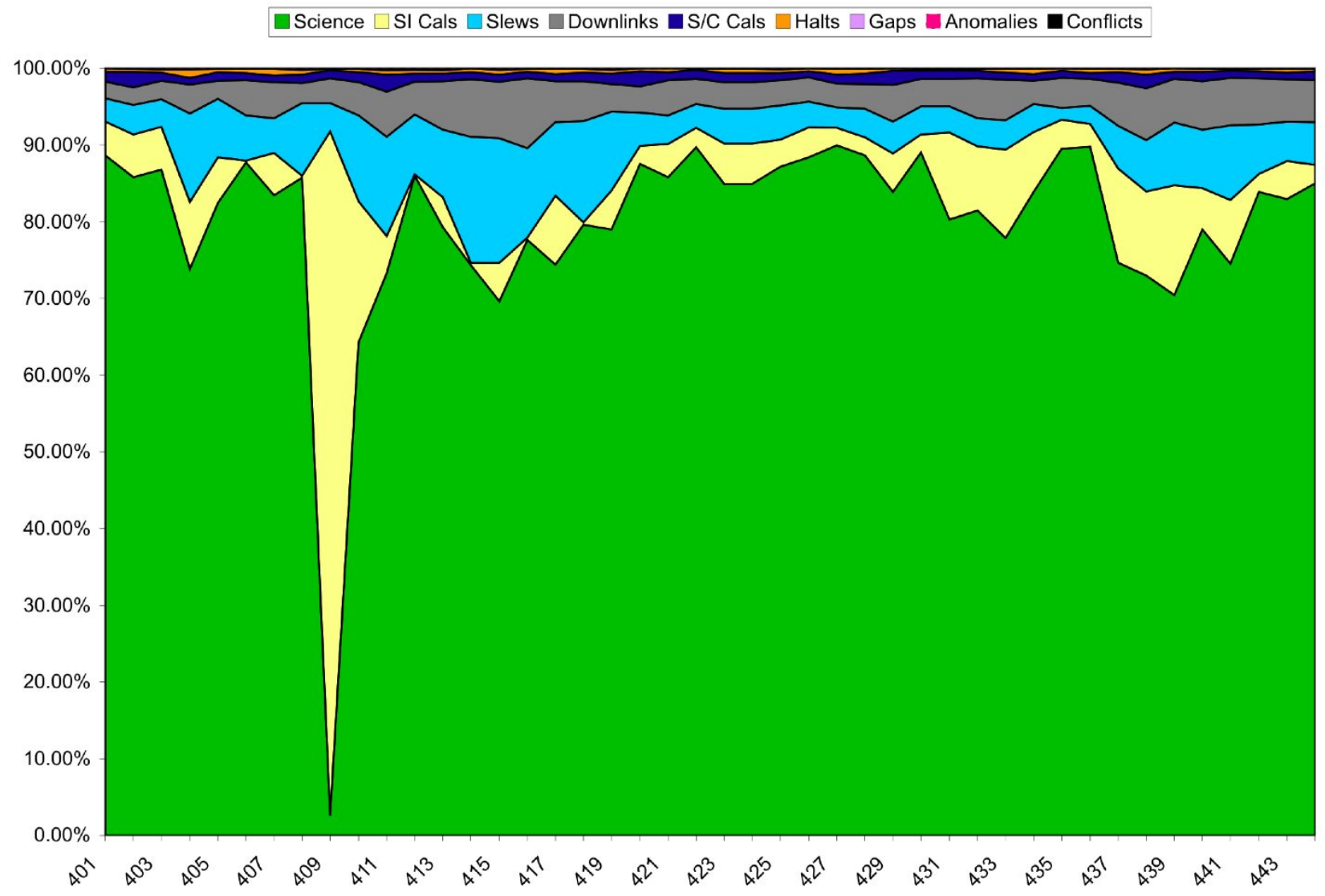

Figure 5. Weekly averages of fractional time spent on various activities. The extensive instrument calibration time in Weeks 409 and 410 correspond to the re-calibration of the IRAC instrument at $28.7 \mathrm{~K}$.

\subsection{Warm Mission Unique Challenges}

The selected warm mission science places great emphasis in exo-planet observations, especially those in excess of 24 hours. In addition to needing special DSN tracks, the long observations require special coordination with the spacecraft operations team to properly schedule spacecraft activities. Preceding the observation we must usually schedule a forced momentum de-saturation and extend the command loss timer setting. Following the long observation, we must add several PCRS/star tracker calibrations to make up for those missed.

Perhaps the biggest new challenge for scheduling the warm mission is obtaining sufficient DSN coverage to support the complex observational suite. As Spitzer recedes from Earth, the strength of the signal received from the observatory continues to decrease (Figure 6). The current maximum downlink rate is $1.65 \mathrm{Mbps}$, but even under the best conditions it will drop to $1.1 \mathrm{Mbps}$ by the fall of 2010 and to $550 \mathrm{kbps}$ by the fall of 2012 (Table 3). As the rates are reduced, the downlink durations will need to be increased proportionally cutting into the overall efficiency.

Spitzer's X-band telecommunication system is fully redundant and has operated without issue. Throughout the prime mission, Spitzer has used a single 15-W solid-state power amplifier (SSPA) for data transmission. The observatory has the capability of transmission powered by dual SSPAs that would double the radiated power and greatly extend the cutoff times for higher data rates. Should the project decide any added risk is acceptable, use of dual SSPAs would both decrease the demands on the DSN resources and would significantly increase efficiency.

Because Spitzer is now in an extended mission phase, its priority for DSN allocations has dropped below that of other missions. Furthermore, its relatively high data rate and weak signal now dictate use of the DSN 70-meter antennae of which there is only one at each of the three DSN complexes (Goldstone, California; Canberra, Australia; and Madrid, 
Spain). The 70-meter antenna is generally arrayed with a 34-meter antenna to boost performance. However, competition for time with other projects, especially Mars projects when that planet is in the same region of sky as Spitzer, together with antenna maintenance and repairs (the Goldstone 70-meter antenna is down for more than half of 2010 for extended servicing), makes it difficult to obtain favorable allocations. The Spitzer DSN scheduling goal is one 3-hour 70-meter/34-meter array track per day with elevation angles above 30 degrees. However, because of the temporary loss of the Goldstone 70-meter antenna and because of strong competition, many of our tracks are not meeting these criteria (e.g. stand alone 70-meter antenna, low elevation angles, less than 3-hour duration). The majority of the recent downlink rates have been dropped to $1.1 \mathrm{Mbps}$ with an occasional 48-hour PAO necessitated by an unscheduled track. Furthermore it is becoming increasingly difficult to obtain tracks consistent with scheduling the long (>60 hours) exo-planet observations. These problems not only complicate scheduling and delay some observations, but they also increase the workload on the Flight Control Team. Because of the reduced staffing level, there are also fewer highimpact ToOs allowed, nominally one per year (for implementation see Tyler et al. ${ }^{[10]}$ ).

Table 3: Cutoff dates for downlinks at the allowed rates using either a single SSPA or dual SSPAs. The rates are listed for both a standalone 70-meter DSN antenna and a 70-meter/34-meter array assuming an elevation angle above 30 degrees under normal weather conditions. With a single SSPA, the current maximum rate is $1.65 \mathrm{Mbps}$.

\begin{tabular}{|c|c|c|c|c|}
\hline \multirow{2}{*}{ Downlink Rate } & \multicolumn{2}{|c|}{ Single SSPA } & \multicolumn{2}{c|}{ Dual SSPA } \\
\cline { 2 - 5 } & Single 70m & 70m/34m Array & Single 70m & 70m/34m Array \\
\hline $2.2 \mathrm{Mbps}$ & 2009 Jan 13 & 2009 Sep 30 & 2011 Jan 22 & 2011 Nov 9 \\
\hline $1.65 \mathrm{Mbps}$ & 2010 Jan 14 & 2010 Oct 17 & 2012 Nov 13 & $2013 \mathrm{Feb} \mathrm{1}$ \\
\hline $1.1 \mathrm{Mbps}$ & 2011 Dec 8 & 2012 Sep 18 & Beyond 2014 & Beyond 2014 \\
\hline $550 \mathrm{kbps}$ & Beyond 2014 & Beyond 2014 & Beyond 2014 & Beyond 2014 \\
\hline $275 \mathrm{kbps}$ & Beyond 2014 & Beyond 2014 & Beyond 2014 & Beyond 2014 \\
\hline
\end{tabular}

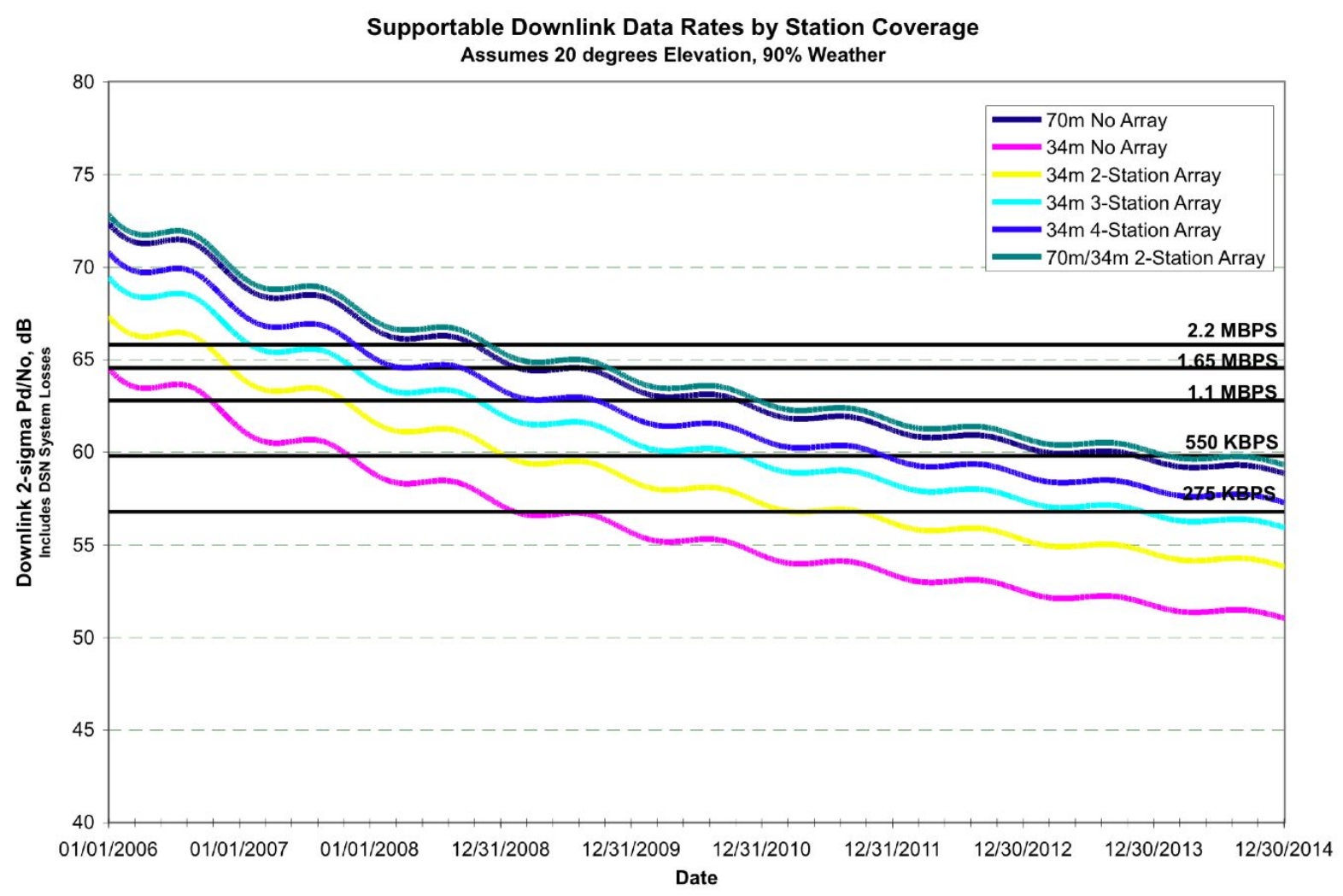

Figure 6. Maximum downlink rates as a function of time for various DSN station configurations. The current maximum downlink rate is $1.65 \mathrm{Mbps}$ but non-optimal DSN tracks often reduce it to $1.1 \mathrm{Mbps}$. 


\section{SUMMARY AND CONCLUSIONS}

Spitzer completed nearly $5 \frac{1}{2}$ years of science observations by the time the cryogen was depleted on 15 May 2009 . Scheduling has been exceptionally efficient with nearly $90 \%$ of the time devoted to science when averaged over the mission lifetime, even accounting for time lost to anomalies. During the prime mission, over 36,000 hours of science observations were executed. This superb efficiency has been possible because of the skill and dedication of the members of the operations teams despite a growing number of scheduling challenges and constraints.

Following the exhaustion of helium, Spitzer entered the IWIC phase that was slated for completion within six weeks in order to expeditiously return to science operations. However, an anomaly in the IRAC array temperature control system caused a one-month delay in the start of IWIC. By taking advantage of advance planning and the pre-built minisequences, the complexity of re-planning IWIC was reduced and the original goals were successfully completed.

The limited on-board data storage capacity continues to be a major scheduling driver in the warm mission and in a number of cases it has dictated the science content of a sequence. It also increases the risk of lost data and complicates telecommunications by requiring special flight control operations to manage the MMC.

Because of the great distance to Spitzer, the project is now entirely dependent on the three DSN 70-meter antennae for receipt of data and it must compete with numerous other operating missions for available time. As the observatory continues to recede from the Earth, the downlink data rates will need to drop from the current maximum of $1.65 \mathrm{Mbps}$ to $550 \mathrm{kbps}$ later in the warm mission. The downlink durations will increase proportionally. Should the project decide to use the dual SSPA capability, the higher downlink rates could be maintained longer with a significant increase in overall efficiency and in reduced demands for DSN time.

During an extended warm mission the scheduling and sequencing processes remain the same as for the prime mission. While only one instrument is used, IRAC has historically been the most difficult to schedule because of tight constraints and high data volumes. These problems will all remain along with more difficult telecommunication restrictions with fewer DSN tracks and a decreasing signal level. To continue operating the mission with a reduced staff will require some loss in observational efficiency and less flexibility in accommodating complex observations, late AOR changes, or ToOs. Nevertheless, should the full extended mission be approved, Spitzer will return an additional 30,000 - 35,000 hours of cutting edge science dominated by large Exploration Science programs.

Acknowledgment: The research described in this paper was carried out at the Jet Propulsion Laboratory and the Spitzer Science Center, California Institute of Technology, under a contract with the National Aeronautics and Space Administration. The authors wish to thank Paul Finley, Mark Garcia, and Shanti Ancheta for assisting with the graphics.

\section{REFERENCES}

[1] Werner, M. W., el al. "The Spitzer Space Telescope Mission,” ApJS, 154, 1 (2004).

[2] Kwok, J. H. "A New Mission Concept for the Space Infrared Telescope Facility," in AAS/AIAA Astrodynamics Conference, Aug. 16-19, 1993, V. J. Modi et al. eds., 1401 (1993).

[3] Sarrel, M. A., and Hunt, J. C., Jr., "Evaluating Requirements on the Spitzer Mission Operations System Based on Flight Operations Experience," Proc. SPIE 7016, 70161P (2008).

[4] Hunt, J., C., Jr., Sarrel, M. A., and Mahoney, W. A. "Spitzer Mission Operations Planning for IRAC WarmInstrument Characterization," SpaceOps, submitted (2010).

[5] Carey, S., et al., "Calibration and data quality of warm IRAC," this meeting, Conference 7731, submitted (2010)

[6] Mahoney, W., et al. "Spitzer Scheduling Challenges: cold and warm," Proc. SPIE 7016, 70161W (2008).

[7] Storrie-Lombardi, L., and Silbermann, N., eds. [The Science Opportunities for the Warm Spitzer Mission Workshop], American Institute of Physics, New York (2007).

[8] Barba, S. J., Garcia, L. J., McElroy, D. B., Mittman, D. S., O'Linger, J. C., and Tyler, S. R. "Planning and Scheduling the Spitzer Space Telescope," Proc. SPIE, 6270, pp. 62700Z (2006).

[9] Sarrel, M. A., Carrion, C., and Hunt, J. C., Jr., "Managing the On-board Data Storage, Acknowledgement and Retransmission System for Spitzer," AIAA SpaceOps 2006, 5564, 1 (2006)

[10] Tyler, S. et al. "Rapid replacement of Spitzer Space Telescope sequences: targets of opportunity and anomalies," Proc. SPIE 7016, 70161V (2008). 